\title{
Prominent Role of Spin-Orbit Coupling in FeSe Revealed by Inelastic Neutron Scattering
}

\author{
Mingwei Ma, ${ }^{1}$ Philippe Bourges, ${ }^{2}$ Yvan Sidis, ${ }^{2}$ Yang Xu, ${ }^{3}$ Shiyan Li,${ }^{3,4}$ Biaoyan $\mathrm{Hu},{ }^{1}$ \\ Jiarui $\mathrm{Li},{ }^{1} \mathrm{Fa}$ Wang, ${ }^{1,5}$ and Yuan $\mathrm{Li}^{1,5, *}$ \\ ${ }^{1}$ International Center for Quantum Materials, School of Physics, Peking University, Beijing 100871, China \\ ${ }^{2}$ Laboratoire Léon Brillouin, CEA-CNRS, Université Paris-Saclay, CEA Saclay, \\ 91191 Gif-sur-Yvette, France \\ ${ }^{3}$ State Key Laboratory of Surface Physics, Department of Physics, and Laboratory of Advanced Materials, \\ Fudan University, Shanghai 200433, China \\ ${ }^{4}$ Collaborative Innovation Center of Advanced Microstructures, Nanjing 210093, China \\ ${ }^{5}$ Collaborative Innovation Center of Quantum Matter, Beijing 100871, China
}

(Received 14 October 2016; revised manuscript received 17 March 2017; published 18 May 2017)

\begin{abstract}
In most existing theories for iron-based superconductors, spin-orbit coupling (SOC) has been assumed to be insignificant. Here, we use spin-polarized inelastic neutron scattering to show that collective low-energy spin excitations in the orthorhombic (or "nematic") phase of FeSe possess nearly no in-plane component. Such spin-space anisotropy is present over an energy range greater than the superconducting gap $2 \Delta_{\mathrm{sc}}$ and gets fully inherited in the superconducting state, resulting in a $c$-axis polarized "spin resonance" without any noticeable isotropic spectral-weight rearrangement related to the superconductivity, which is distinct from observations in the superconducting iron pnictides. The contrast between the strong suppression of long-range magnetic order in FeSe and the persisting large spin-space anisotropy, which cannot be explained microscopically by introducing single-ion anisotropy into local-moment spin models, demonstrates the importance of SOC in an itinerant-electron description of the low-energy spin excitations. Our result helps to elucidate the nearby magnetic instabilities and the debated interplay between spin and orbital degrees of freedom in FeSe. The prominent role of SOC also implies a possible unusual nature of the superconducting state.
\end{abstract}

DOI: 10.1103/PhysRevX.7.021025

\author{
Subject Areas: Magnetism, \\ Strongly Correlated Materials, \\ Superconductivity
}

Spin-orbit coupling (SOC) is a fundamental interaction in solids due to the relativistic motion of electrons. The effect of SOC is expected to be pronounced in the presence of heavy elements, whereas its relevance to the iron-based superconductors has thus far been assumed insignificant in most theoretical treatment. This assumption has led to a separation of ideas, in particular, those attempting to explain the formation of superconductivity [1-3] and nematic order [4-10] into "spin" and "orbital" camps. Indeed, in the presence of SOC, neither the spin nor the orbital angular momentum remains a good quantum number; hence, any collective electronic behavior would have to be considered as a consequence of the joint interactions involving both.

Recent angle-resolved photoemission spectroscopy (ARPES) experiments have suggested the presence of

\footnotetext{
*yuan.li@pku.edu.cn
}

Published by the American Physical Society under the terms of the Creative Commons Attribution 4.0 International license. Further distribution of this work must maintain attribution to the author(s) and the published article's title, journal citation, and DOI.
SOC in iron-based superconductors, via the observation of electronic band splitting at the Brillouin zone (BZ) center in the tetragonal phase [11-13]. The affected quasiparticle states can be expected to have spin structures that will give rise to spin-space anisotropy (SSA) in the magnetism, if the essence of the magnetism can be captured by an itinerant (spin-density-wave-like) description [14]. Indeed, an energy gap related to SSA has been observed by inelastic neutron scattering (INS) in pnictide parent compounds [15] with long-range stripe-antiferromagnetic order. Spin-polarized INS experiments [16-18] have further shown that the SSA is " $X Y Z$ " (orthorhombic) rather than " $X X Z$ " (tetragonal), which suggests that orbital polarization in the nematic phase might be its origin [16]. However, as we discuss later, single-ion anisotropy $[16,17]$ in local-moment spin models for the FeAs layers qualitatively fails to explain the observed SSA, and orbital polarization is generically expected to suppress the SSA. Thus, the microscopic origin of the SSA was simply unknown. Another limitation of the pnictides is that the nematic order is closely accompanied by magnetic order, so it remains unclear whether the SSA depends on the presence of the nearby magnetic order. In order to assess the influence of SOC on the magnetism at a more 

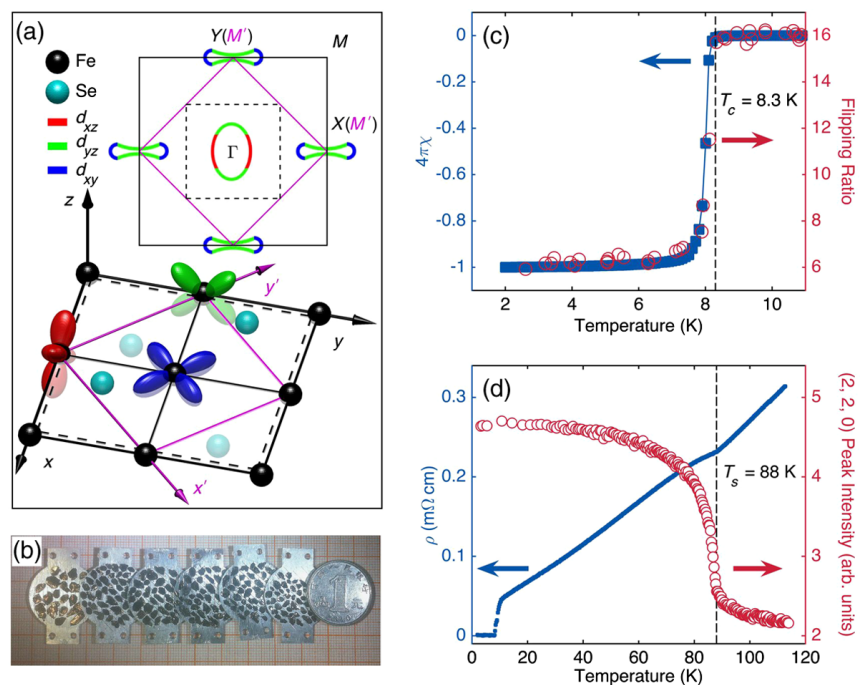

FIG. 1. Crystal structure, FS topology, and characterization of phase transition temperatures in FeSe single crystals. (a) Crystal structure and FS topology [20], with orbital characters color coded with the orbital shapes. Three Brillouin zone notations are shown for comparison: 1-Fe unit cell (black solid lines), 2-Fe unit cell (magenta solid lines), and 4-Fe unit cell (dashed lines, used throughout our presentation). (b) Photograph of our INS sample before the final assembly. (c) dc magnetic susceptibility $\chi$ shows a sharp superconducting transition at $T_{c}=8.3 \mathrm{~K}$ in zero-fieldcooled measurement in a magnetic field of $\mathrm{H}=10 \mathrm{Oe}$ applied parallel to the $a b$ plane. A consistent transition is observed for the entire INS sample using depolarization effect on the neutron beam. (d) Resistivity $\rho$ shows an abrupt change in slope versus $T$ at the nematic transition temperature $T_{s}=88 \mathrm{~K}$, below which an abrupt increase in the $(2,2,0)$ Bragg scattering intensity is observed due to reduced extinction effects.

fundamental level, in particular, to test the validity of itinerant descriptions of the magnetism in conjunction with our knowledge on the electronic structure, it is highly desirable to study SSA in a system that is relatively far from developing long-range magnetic order.

FeSe offers an ideal opportunity for this purpose. As structurally the simplest Fe-based superconductor, FeSe has nematic order below $T_{s} \approx 90 \mathrm{~K}$ but no magnetic order down to the lowest temperature [19], featuring the largest separation between $T_{s}$ and the Néel temperature $T_{N}$. This large separation has motivated researchers to argue that magnetism is inessential for the formation of the nematic order $[6,10]$. If the SSA observed in the pnictides is indeed associated with stripe antiferromagnetism, which selects an in-plane spin direction for the order itself and leaves the remaining two directions permissible for spin-wave-like excitations, it is expected to be weaker or different in FeSe. Figure 1(a) displays the crystal and Fermi surface (FS) structure of FeSe in the nematic phase [20], along with leading $\mathrm{Fe} 3 d$ orbital characters ( $x y, x z$, and $y z$ ) of quasiparticle states on the FS. Throughout this paper, we use the 4-Fe unit cell, for which the BZ is shown by the dashed lines in Fig. 1(a). The centers of the hole and electron pockets are connected by two-dimensional wave vectors $(1,0)$ and $(0,1)$ in reciprocal lattice units (r.l.u.).

Our spin-polarized INS experiment is carried out on the 4F1 triple-axis spectrometer at the Laboratoire Léon Brillouin, Saclay, France (the instrument configurations are described in the Supplemental Material [21]). Over 400 single crystals of FeSe [Fig. 1(b)] are used for this study, with a total mass of about $3.5 \mathrm{~g}$. They are grown with a chemical-vapor-transport method and coaligned within $6^{\circ}$ mosaicity in the $(H, K, 0)$ scattering plane on aluminum plates using a hydrogen-free adhesive. Because of the heavy loss in incident flux and detection efficiency associated with current polarized-neutron techniques, very long measurement time ( $3 \mathrm{~h}$ or more per data point) is required to acquire satisfactory statistical accuracy. The quality of spin polarization in a neutron scattering experiment can be quantified by the flipping ratio, defined here as the ratio between intensities measured in non-spin-flip (NSF) and spin-flip geometries on strong nuclear Bragg peaks. The flipping ratio in our experiment is about 16 for all neutron spin polarizations [Fig. 1(c)]. Because the spin polarization is maintained by a guide magnetic field $(\sim 10 \mathrm{Oe})$ throughout the beam path, the beam can be partially depolarized if the guide field changes abruptly, such as at the sample surface when the sample is in a diamagnetic (Meissner) state below $T_{c}$. This offers a method to measure $T_{c}$ for the entire sample array. To do this, the sample is cooled below $T_{c}$ in a guide field so that it contains trapped vortices, and then the guide field direction is rotated by $90^{\circ}$. The flipping ratio is reduced to $\sim 6$ after the guide-field rotation and is measured upon warming up the sample [Fig. 1(c)], with a recovery to its original value at $T_{c}$. The superconducting critical temperature, $T_{c} \approx 8.3 \mathrm{~K}$ [Fig. 1(c)], and the nematic transition temperature, $T_{s} \approx 88 \mathrm{~K}$ [Fig. 1(d)], are determined on selected crystals by magnetic susceptibility and resistivity measurements, respectively, as well as on the entire sample by neutron scattering methods.

Previous unpolarized INS studies on FeSe $[4,22]$ have uncovered strong magnetic signals at $\mathbf{Q}=(1,0,0)$ below $T_{s}$ and a spin resonance at $4 \mathrm{meV}$ below $T_{c}$. For INS samples which are typically twinned, the same scattering signal can be expected at $\mathbf{Q}=(0,1,0)$, as depicted in Fig. 2(a). For convenience, we keep the notation $\mathbf{Q}=$ $(1,0,0)$, and the nominal $b$ direction should be understood as within the FeSe plane and perpendicular to Q. As illustrated in Fig. 2(b), INS detects only spin fluctuations in directions perpendicular to $\mathbf{Q}$, i.e., along $b\left(M_{b}\right)$ and $c\left(M_{c}\right)$ for $\mathbf{Q}=(1,0,0)$. By analyzing the neutron-spin dependence of the scattering signal, we are able to determine these two components separately. Most of our data are obtained in spin-flip channels with the incoming neutron spin along $\mathbf{Q}\left(\mathrm{SF}_{\mathbf{Q}}\right), b\left(\mathrm{SF}_{b}\right)$, and $c\left(\mathrm{SF}_{c}\right)$ directions, which detect the $M_{b}+M_{c}, M_{c}$, and $M_{b}$ components, respectively, on top of a common background (BG). 



FIG. 2. Spin-polarized INS selection rules and predominant $M_{c}$ contribution to the magnetic signal. (a) Distribution of low-energy magnetic signals in momentum space. (b) Total spin fluctuations $\left(M_{\text {total }}\right)$ and its three components, two of which can be detected by INS at $\mathbf{Q}=(1,0,0)$ with selection rules described in the text. (c) Momentum scans at fixed energies $(E)$ along a trajectory shown in (a). (d) Energy scans at fixed $\mathbf{Q}=(1,0,0)$. Panels (c) and (d) share the same legends that indicate the three spin-flip channels. The two sets of symbols in (d) represent data obtained with different sample-environment devices. The BG intensity is determined from the selection rule: $\mathrm{BG}=\mathrm{SF}_{b}+$ $\mathrm{SF}_{c}-\mathrm{SF}_{\mathbf{Q}}$, taking all available data points into account (see Supplemental Material [21]). Shaded area in (d) indicates statistical uncertainty of our BG determination.

Figure 2(c) displays constant-energy scans at 2.5 and $4.0 \mathrm{meV}$, performed at $10 \mathrm{~K}$ along a trajectory shown in Fig. 2(a). A clear commensurate peak is seen in the $\mathrm{SF}_{\mathbf{Q}}$ channel, consistent with the previous unpolarized INS results [4]. If the scattering is isotropic in spin space, the signal should be equally distributed in the $\mathrm{SF}_{b}$ and $\mathrm{SF}_{c}$ channels, which is clearly not the case. By comparing data obtained in the three different channels, we are able to determine the nonmagnetic BG intensity (see Supplemental Material [21]), which in some cases is higher in the middle of the scan because the magnitude of $\mathbf{Q}$ is shortest there. Figure 2(d) displays the energy-dependent BG together with raw data from energy scans measured at fixed $\mathbf{Q}=(1,0,0)$. We find that the intensities in the $\mathrm{SF}_{\mathbf{Q}}$ and $\mathrm{SF}_{b}$ channels are nearly equal over the entire measured energy range $2.5 \leq E \leq 8 \mathrm{meV}$. This means that the $\mathrm{SF}_{c}$ intensity is mostly $\mathrm{BG}$, and that the magnetic signal is dominated by its $M_{c}$ component.

By measuring only at $\mathbf{Q}=(1,0,0)$, we are nominally not sensitive to the $M_{a}$ component [Fig. 2(b)]. For a twinned sample below $T_{s}$, however, we simultaneously detect magnetic signals from the two nematic domains, which is equivalent to detecting physical signals from both $\mathbf{Q}_{1}=(1,0,0)$ and $\mathbf{Q}_{2}=(0,1,0)$, with $\left\{M_{b}, M_{c}\right\}$ and $\left\{M_{a}, M_{c}\right\}$ components, respectively. Our data show that $M_{b}\left(\mathbf{Q}_{1}\right)+M_{a}\left(\mathbf{Q}_{2}\right)$ is negligible compared to $M_{c}\left(\mathbf{Q}_{1}\right)+$ $M_{c}\left(\mathbf{Q}_{2}\right)$. If low-energy spin excitations are controlled by FS nesting [14], we believe that the overall intensity


FIG. 3. Evolution of different spin-fluctuation components with temperature. (a)-(c) Net magnetic signal components at $\mathbf{Q}=$ $(1,0,0)$ at three different temperatures, obtained by subtracting the globally determined BG intensity (see Fig. 2 and Supplemental Material [21]) from $\mathrm{SF}_{\mathbf{Q}}, \mathrm{SF}_{b}$, and $\mathrm{SF}_{c}$ data, respectively. (d) Intensity change across $T_{c}$ measured in different geometries. The non-spin-flip scattering geometry with incoming neutron spin polarization along $c\left(\mathrm{NSF}_{c}\right)$ measures spin fluctuations along the $c$ direction and confirms the spin-flip $\left(\mathrm{SF}_{b}\right)$ result. Solid lines are guides to the eye. The two sets of symbols in (a)-(c) for $10 \mathrm{~K}$ represent data obtained with different sample-environment devices (see Supplemental Material [21]).

difference between $\mathbf{Q}_{1}$ and $\mathbf{Q}_{2}$ (in a detwinned sample) is not large, because the orbital characters and quality of FS nesting are very similar [Fig. 1(a)]. Our result, hence, suggests that the low-energy spin excitations have nearly no in-plane components $\left(M_{a b}\right)$.

We present in Fig. 3 the evolution of spin fluctuations with temperature. In the tetragonal phase, the overall intensity is weak and the data are consistent with an isotropic distribution of scattering signals in $M_{b}$ and $M_{c}$. Upon cooling into the nematic phase, a strong enhancement is found in $M_{c}$ [Fig. 3(b)] but not in $M_{b}$ [Fig. 3(c)]. Upon further cooling below $T_{c}$, the magnetic spectral weight rearranges itself into a spin resonance [4] at $4 \mathrm{meV}$. Our data unambiguously show that the resonance is essentially fully $c$-axis polarized. This can be directly seen from the intensity difference between 2 and $10 \mathrm{~K}$, and is also supported by data obtained in the NSF geometry [Fig. 3(d)]: there is a clear intensity change across $T_{c}$ near $4 \mathrm{meV}$ in $M_{c}$ but no change beyond statistical uncertainty in $M_{b}$. To assess the statistical accuracy of our data in the determination of the contrast between $M_{c}$ and $M_{b}$ over the 
TABLE I. Summary of SSA, in particular, the relative strength of $M_{c}$ and $M_{a b}$, in iron pnictides and FeSe. The following acronyms are used in the table: resonance energy $\left(E_{\text {res }}\right)$, nonsuperconducting (non-SC), not applicable (NA), $\left.\mathrm{Ba}_{\left(\mathrm{Fe}_{1-x} \mathrm{Co}\right.}\right)_{2} \mathrm{As} s_{2}(\mathrm{BFCA}$ ), $\mathrm{Ba}\left(\mathrm{Fe}_{1-x} \mathrm{Ni}_{x}\right)_{2} \mathrm{As}_{2}$ (BFNA), $\mathrm{NaFe}_{1-x} \mathrm{Co}_{x} \mathrm{As}$ (NFCA), $\mathrm{Ba}_{1-x} \mathrm{~K}_{x} \mathrm{Fe}_{2} \mathrm{As}_{2}$ (BKFA), underdoped (UD), optimally doped (OP), overdoped (OV). Results for the pnictides are based on measurements near the momentum transfer $(1,0,1)_{o}$ (orthorhombic notation) or equivalent positions that correspond to the magnetic wave vector in the parent compound. In some of these measurements [24,26-28], no attempt was made to separately determine $M_{c}$ and $M_{a}$, in which cases the ratio of $M_{c} / M_{a b}$ may have been overestimated. We use bold notation to indicate the energy of the main spin resonance in the pnictides and the fact that the corresponding INS signal is isotropic in spin space.

\begin{tabular}{|c|c|c|c|c|c|c|c|c|}
\hline Compound & Doping & $\begin{array}{c}T_{s}, T_{N} \\
\quad \text { and } \\
T_{c}(\mathrm{~K})\end{array}$ & $\begin{array}{c}E_{\mathrm{res}} \\
(\mathrm{meV})\end{array}$ & $\begin{array}{c}\text { SSA energy } \\
\text { range } \\
(\mathrm{meV})\end{array}$ & $\begin{array}{l}\text { Representative } \\
M_{c} / M_{a b}, \\
T \gtrsim T_{c}\end{array}$ & $\begin{array}{c}\text { Representative } \\
M_{c} / M_{a b}, \\
T \ll T_{c}\end{array}$ & Notes & References \\
\hline $\mathrm{BaFe}_{2} \mathrm{As}_{2}$ & Parent & $\begin{array}{c}137,137 \\
\text { non-SC }\end{array}$ & NA & $<18$ & 10 at $12 \mathrm{meV}$ & NA & $M_{a b}$ is mostly $M_{b}$ & {$[16,17]$} \\
\hline $\mathrm{NaFeAs}$ & Parent & $\begin{array}{c}58,45 \\
\text { non-SC }\end{array}$ & NA & $<13$ & 10 at $8 \mathrm{meV}$ & NA & $M_{a b}$ is mostly $M_{b}$ & {$[18]$} \\
\hline $\begin{array}{l}\text { BFCA } \\
x=0.045\end{array}$ & $\begin{array}{l}n \text {-type } \\
\text { UD }\end{array}$ & $\begin{array}{c}65,55 \\
14\end{array}$ & $4.7, \mathbf{5 . 9}$ & $<12$ & 2 at $7 \mathrm{meV}$ & $\begin{array}{c}\mathbf{1} \text { at } 5.9 \mathrm{meV} \\
2.5 \text { at } 4.7 \mathrm{meV}\end{array}$ & $M_{a b}$ is mostly $M_{b}$ & [29] \\
\hline $\begin{array}{l}\text { BFNA } \\
x=0.048\end{array}$ & $\begin{array}{l}n \text {-type } \\
\text { UD }\end{array}$ & $\begin{array}{c}33,33 \\
20\end{array}$ & 7.0 & $<7$ & 1 at $3 \mathrm{meV}$ & $\begin{array}{l}\mathbf{1} \text { at } 7 \mathrm{meV} \\
\mathbf{1} \text { at } 3 \mathrm{meV}\end{array}$ & $M_{a b}$ is mostly $M_{a}$ & [23] \\
\hline $\begin{array}{l}\text { NFCA } \\
x=0.015\end{array}$ & $\begin{array}{l}n \text {-type } \\
\text { UD }\end{array}$ & $\begin{array}{c}35,30 \\
15\end{array}$ & $4.0,7.0$ & $<7$ & 4 at $4 \mathrm{meV}$ & $\begin{array}{l}\mathbf{1} \text { at } 7 \mathbf{m e V} \\
1.5 \text { at } 4 \mathrm{meV}\end{array}$ & $M_{a b}$ is mostly $M_{a}$ & {$[25,30]$} \\
\hline $\begin{array}{l}\text { BFCA } \\
x=0.06\end{array}$ & $\begin{array}{l}n \text {-type } \\
\text { OP }\end{array}$ & $\begin{array}{c}\text { NA, NA } \\
24\end{array}$ & $4.0, \mathbf{8 . 0}$ & $<6$ & 2 at $4 \mathrm{meV}$ & $\begin{array}{l}\mathbf{1} \text { at } 8 \mathbf{~ m e V} \\
20 \text { at } 4 \mathrm{meV}\end{array}$ & $\begin{array}{l}\operatorname{mixed} M_{c} \text { and } M_{a} \\
\quad \text { at } \mathbf{Q}=(1,0,1)_{o}\end{array}$ & [24] \\
\hline $\begin{array}{l}\text { BFNA } \\
x=0.05\end{array}$ & $\begin{array}{l}n \text {-type } \\
\text { OP }\end{array}$ & $\begin{array}{c}\text { NA, NA } \\
20\end{array}$ & 7.0 & $<5$ & 1.5 at $3 \mathrm{meV}$ & $\begin{array}{l}\mathbf{1} \text { at } 7 \mathrm{meV} \\
3 \text { at } 3 \mathrm{meV}\end{array}$ & $\begin{array}{l}\operatorname{mixed} M_{c} \text { and } M_{a} \\
\quad \text { at } \mathbf{Q}=(1,0,1)_{o}\end{array}$ & [26] \\
\hline $\begin{array}{l}\text { BKFA } \\
x=0.33\end{array}$ & $\begin{array}{l}p \text {-type } \\
\text { OP }\end{array}$ & $\begin{array}{l}\text { NA, NA } \\
38\end{array}$ & 15 & $<15$ & 3 at $3 \mathrm{meV}$ & $\begin{array}{c}\mathbf{1} \text { at } \mathbf{1 5} \mathbf{~ m e V} \\
4 \text { at } 7 \mathrm{meV}\end{array}$ & $\begin{array}{l}\operatorname{mixed} M_{c} \text { and } M_{a} \\
\quad \text { at } \mathbf{Q}=(1,0,1)_{o}\end{array}$ & [27] \\
\hline $\begin{array}{l}\text { BKFA } \\
x=0.50\end{array}$ & $\begin{array}{l}p \text {-type } \\
\text { OV }\end{array}$ & $\begin{array}{l}\text { NA, NA } \\
36\end{array}$ & 15 & $<15$ & 1 & $\begin{array}{l}\mathbf{1} \text { at } \mathbf{1 5} \mathbf{~ m e V} \\
2 \text { at } 12 \mathrm{meV}\end{array}$ & $\begin{array}{l}\operatorname{mixed} M_{c} \text { and } M_{a} \\
\quad \text { at } \mathbf{Q}=(1,0,1)_{o}\end{array}$ & [28] \\
\hline $\begin{array}{l}\text { NFCA } \\
x=0.045\end{array}$ & $\begin{array}{l}n \text {-type } \\
\text { OV }\end{array}$ & $\begin{array}{c}\text { NA, NA } \\
18\end{array}$ & 7.0 & NA & 1 & 1 & no SSA & {$[25]$} \\
\hline $\begin{array}{l}\text { BFNA } \\
x=0.075\end{array}$ & $\begin{array}{l}n \text {-type } \\
\text { OV }\end{array}$ & $\begin{array}{c}\text { NA, NA } \\
14\end{array}$ & 7.0 & NA & 1 & 1 & no SSA & {$[31]$} \\
\hline $\mathrm{FeSe}$ & Pristine & $\begin{array}{c}88, \text { NA } \\
8.3\end{array}$ & 4.0 & at least up to 8 & $7.3 \pm 3.2$ & $7.3 \pm 3.2$ & $\operatorname{mixed} M_{a}$ and $M_{b}$ & This work \\
\hline
\end{tabular}

entire energy range (from 2.5 to $8 \mathrm{meV}$ ), we consider all raw data (with statistical errors) measured at 2 and $10 \mathrm{~K}$ in the $\mathrm{SF}_{\mathbf{Q}}, \mathrm{SF}_{b}$, and $\mathrm{SF}_{c}$ channels. By taking an equalweight average of the data, we obtain $\mathrm{SF}_{\mathbf{Q}}=12.37 \pm 0.17$, $\mathrm{SF}_{b}=11.86 \pm 0.17$, and $\mathrm{SF}_{c}=8.65 \pm 0.14$, from which we infer $M_{c} / M_{b}=7.3 \pm 3.2$ (assumed to be unchanged across $T_{c}$ ).

It is revealing to compare our results to SSA observed in the iron pnictides, which we summarize in Table I with the emphasis of comparing the $M_{c}$ and $M_{a b}$ signals. We begin by noting that, toward the lowest energies, spin excitations in the magnetically ordered parent compounds [16-18] are dominated by $M_{c}$, since $M_{a}$ is forbidden in the spin-wave spectrum, and because $M_{c}$ has a smaller energy gap than $M_{b}$. Using single-ion anisotropy in localmoment spin models to describe the phenomenon, one sees that the sizes of these energy gaps are greatly enlarged from the bare anisotropy energies $\left(D_{\alpha}, \alpha=b, c\right)$ as $\sim \sqrt{D_{\alpha} J}$ [17], where $J\left(\gg D_{\alpha}\right)$ is the strongest exchange interaction. The presence of these large and different energy gaps leads to large $M_{c} / M_{a b}$ ratios measured below the gap of $M_{b}$ but above the gap of $M_{c}$. With increasing doping, the gaps (in the normal state, $T>T_{c}$ ) rapidly decrease and become no longer measurable before [23] or as soon as the magnetic order is completely suppressed [24], meanwhile a nonzero $M_{a}$ component is recovered [23,25], both of which lead to much reduced $M_{c} / M_{a b}$ ratios. Our result for FeSe is rather surprising in this context: Despite the lack of longrange order and gap in $M_{c}$ [down to $2.5 \mathrm{meV}$, Fig. 3(b)], $M_{a b}$ remains very small, as if the signal is gapped out up to at least $8 \mathrm{meV}$. In the single-ion-anisotropy and localmoment language, which we will soon disprove, this points toward exceedingly large bare anisotropy energies in FeSe which strongly favor spin orientation along $c$ in the paramagnetic state. 
The contrast in SSA between FeSe and the pnictides continues into the superconducting state. The most prominent INS feature in superconducting pnictides, the spin resonance, is commonly observed near an energy of about $(4.6 \pm 0.4) k_{B} T_{c}$ [32]. Even though a second "resonance" at lower energy has been uncovered in recent studies [24,33] with contributions primarily from $M_{c}$ [24,29] and $M_{a}$ [25], or more generically, additional $M_{c}$ (and $M_{a}$ ) signals have been found in the superconducting state below the resonance energy [23,26-28], the main resonance feature is always isotropic in spin space (highlighted in boldface in Table I). Therefore, our data demonstrate that FeSe exhibits the most clear-cut SSA on the spin resonance among all iron-based superconductors measured to date, with a single resonance feature that is highly $c$-axis polarized. The SSA observed in the superconducting state of the pnictides has previously been interpreted as related to the nearby stripe-antiferromagnetic order [24,27]. But because the effect in FeSe is even stronger, we argue that it is likely a joint consequence of strong SOC and the unique electronic structure of FeSe: both the Fermi energy [34] and the superconducting gap $2 \Delta_{\text {sc }}$ are small $[13,34,35]$, compared to $8 \mathrm{meV}$ up to which we are able to detect the SSA.

The observed SSA can be qualitatively understood using an itinerant description of the magnetism, with reasoning similar to that for $\mathrm{Sr}_{2} \mathrm{RuO}_{4}$. Low-energy spin fluctuations in $\mathrm{Sr}_{2} \mathrm{RuO}_{4}$ also have a leading $c$-axis component [36], which can be attributed to the ruthenium $d_{x z}$ and $d_{y z}$ orbital character of quasiparticle states that are most involved in FS nesting [37]. The same applies to FeSe: the quasiparticle states closest to the Fermi level near the $\Gamma$ and $M^{\prime}$ points [Fig. 1(a)] are of predominant Fe $d_{x z}$ and $d_{y z}$ orbital character $[20,38,39]$. In the limits of pure $d_{x z} / d_{y z}$ orbital character and strong atomic SOC, the low-energy electronic states around both the hole and electron pockets will be $|x z+i \cdot y z, \uparrow\rangle$ and $|x z-i \cdot y z, \downarrow\rangle$. The matrix elements of in-plane spin components between these states vanish (see Supplemental Material [21]), whereas without SOC the spin excitations are expected to be isotropic. For both FeSe and the pnictides [16,18], it remains a theoretical challenge to explain the fact that SSA becomes more pronounced in the nematic state, in which the splitting between $d_{x z}$ and $d_{y z}$ orbitals is generally expected to weaken the above spinorbital entanglement. One possibility is that nematic splitting helps to bring some of the band tops and/or band bottoms at $\Gamma$ and $M^{\prime}$ closer to the Fermi level. The bottom line is that the nematic order must not fully quench the orbital angular momentum, otherwise the SSA will disappear. Therefore, the perspective that "orthorhombic orbital structure leads to orthorhombic SSA" is an oversimplification. These considerations can be particularly valuable for our understanding of the electronic structure of FeSe near the $M^{\prime}$ point, where interpretations of ARPES data are still controversial $[11,20,38,40]$.
To explore whether the observed SSA can be microscopically understood in the context of single-ion anisotropy, which is commonly introduced as a phenomenological parameter in local-moment spin models [16,17], we examine the origin of single-ion anisotropy and its qualitative preference (between $M_{c}$ and $M_{a b}$ ) using the same orbitals as above. Details of our analysis are provided in the Supplemental Material [21]. It turns out that SOC in conjunction with the $d_{x z} / d_{y z}$ orbitals always produces easy- $a b$-plane anisotropy at the single-ion level (up to second-order perturbation). While such easy- $a b$-plane anisotropy might explain the observation of slightly more in-plane than $c$-axis magnetic response in $\mathrm{FeTe}_{1-x} \mathrm{Se}_{x}$ $(x=0.5$ [41] and 0.4 [42]), it qualitatively fails to explain the SSA observed in FeSe and the pnictides. Nevertheless, we find that the strength of the anisotropy is slightly enhanced upon the lifting of the $d_{x z} / d_{y z}$ degeneracy, which may be related to the fact that stripe-antiferromagnetic order in the pnictides prefers in-plane spin orientation and often develops slightly below $T_{s}$. The somewhat different case of $\mathrm{FeTe}_{1-x} \mathrm{Se}_{x}$ may be related to the important role of $\mathrm{Te}$ in giving rise to the SOC [12] and the associated anisotropy, and more work is required in order to reach a consistent description for all systems. A further precaution is that, here and in the previous discussions of SSA effects in the pnictides using the local-moment description [16,17], no explicit consideration has been devoted to anisotropy energies beyond the single-ion level. Even though the effect of two-ion (or exchange) anisotropy can usually be mapped onto an effective single-ion anisotropy term for the description of spin-1 excitations, it does not have to be easy- $a b$-plane. Nevertheless, it is unlikely to explain our observation in paramagnetic FeSe since a rather large effective single-ion anisotropy energy will be required.

Our result has important implications on the magnetism in iron-based superconductors. Characteristics of lowenergy spin excitations are usually linked to the nature of nearby magnetic instabilities. While spin fluctuations near $(1,0)$ indicate the presence of magnetic interactions in FeSe that are in favor of stripe antiferromagnetism [4,22], the fact that these fluctuations are predominantly $c$-axis oriented implies that the leading magnetic instability would result in moments along the $c$ axis, consistent with a recent observation under pressure [43]. Moreover, since the observed SSA can be qualitatively explained by the orbital structure, which is to a large extent ubiquitous to all ironbased superconductors, our result is consistent with the notion that the spin-reorientation transition in the pnictides $[44,45]$ arises from a competing magnetic instability that requires the presence of SOC $[46,47]$.

The strong SOC not only helps to reconcile the debate on the spin-orbital interplay, e.g., in driving the nematic order in FeSe [5-10], but might also give rise to novel superconductivity $[48,49]$ by mixing the spin-singlet and triplet Cooper-pairing channels [46]. A consequence of 
such mixture is that Cooper pairs become more robust against applied magnetic fields [49]. We confirm an earlier report [50] of an in-plane upper critical field $H_{c 2}$ in FeSe as large as $27 \mathrm{~T}$ (see Supplemental Material [21]), which exceeds or approaches the Pauli limit $H_{p}=1.414 \Delta_{\mathrm{sc}} / g \mu_{B}$ ( $g$ is Landé $g$ factor, taken to be 2 here, and $\mu_{B}$ is the Bohr magneton), which amounts to $15.9-30.5 \mathrm{~T}$ for reported $\Delta_{\mathrm{sc}}$ values ranging from 1.3 to $2.5 \mathrm{meV}[13,34,35]$.

We wish to thank Jitae Park, Yan Zhang, Weiqiang Yu, Tao Li, Gang Chen, Pengcheng Dai, and Haihu Wen for discussions. This work is supported by the National Natural Science Foundation of China (Grants No. 11374024 and No. 11522429) and Ministry of Science and Technology of China (Grants No. 2015CB921302 and No. 2013CB921903). The work of F. W. was initiated at the Aspen Center for Physics, which is supported by National Science Foundation Grant No. PHY-1066293, and partially supported by a grant from the Simons Foundation.

[1] P. J. Hirschfeld, M. M. Korshunov, and I. I. Mazin, Gap Symmetry and Structure of Fe-Based Superconductors, Rep. Prog. Phys. 74, 124508 (2011).

[2] D. J. Scalapino, A Common Thread: The Pairing Interaction for Unconventional Superconductors, Rev. Mod. Phys. 84, 1383 (2012).

[3] H. Kontani and S. Onari, Orbital-Fluctuation-Mediated Superconductivity in Iron Pnictides: Analysis of the FiveOrbital Hubbard-Holstein Model, Phys. Rev. Lett. 104, 157001 (2010).

[4] Q. Wang, Y. Shen, B. Pan, Y. Hao, M. Ma, F. Zhou, P. Steffens, K. Schmalzl, T. R. Forrest, M. Abdel-Hafiez, X. Chen, D. A. Chareev, A. N. Vasiliev, P. Bourges, Y. Sidis, H. Cao, and J. Zhao, Strong Interplay between Stripe Spin Fluctuations, Nematicity and Superconductivity in FeSe, Nat. Mater. 15, 159 (2016).

[5] R. M. Fernandes, A. V. Chubukov, and J. Schmalian, What Drives Nematic Order in Iron-Based Superconductors?, Nat. Phys. 10, 97 (2014).

[6] S.-H. Baek, D. V. Efremov, J. M. Ok, J. S. Kim, J. van den Brink, and B. Büchner, Orbital-Driven Nematicity in FeSe, Nat. Mater. 14, 210 (2015).

[7] F. Wang, S. A. Kivelson, and D.-H. Lee, Nematicity and Quantum Paramagnetism in FeSe, Nat. Phys. 11, 959 (2015).

[8] J. K. Glasbrenner, I. I. Mazin, H. O. Jeschke, P. J. Hirschfeld, R. M. Fernandes, and R. Valentí, Effect of Magnetic Frustration on Nematicity and Superconductivity in Iron Chalcogenides, Nat. Phys. 11, 953 (2015).

[9] R. Yu and Q. Si, Antiferroquadrupolar and Ising-Nematic Orders of a Frustrated Bilinear-Biquadratic Heisenberg Model and Implications for the Magnetism of FeSe, Phys. Rev. Lett. 115, 116401 (2015).

[10] Y. Yamakawa, S. Onari, and H. Kontani, Nematicity and Magnetism in FeSe and Other Families of Fe-Based Superconductors, Phys. Rev. X 6, 021032 (2016).
[11] M. D. Watson, T. K. Kim, A. A. Haghighirad, N. R. Davies, A. McCollam, A. Narayanan, S. F. Blake, Y. L. Chen, S. Ghannadzadeh, A. J. Schofield, M. Hoesch, C. Meingast, T. Wolf, and A. I. Coldea, Emergence of the Nematic Electronic State in FeSe, Phys. Rev. B 91, 155106 (2015).

[12] P. D. Johnson, H.-B. Yang, J. D. Rameau, G. D. Gu, Z.-H. Pan, T. Valla, M. Weinert, and A. V. Fedorov, SpinOrbit Interactions and the Nematicity Observed in the FeBased Superconductors, Phys. Rev. Lett. 114, 167001 (2015).

[13] S. V. Borisenko, D. V. Evtushinsky, Z.-H. Liu, I. Morozov, R. Kappenberger, S. Wurmehl, B. Büchner, A. N. Yaresko, T. K. Kim, M. Hoesch, T. Wolf, and N. D. Zhigadlo, Direct Observation of Spin-Orbit Coupling in Iron-Based Superconductors, Nat. Phys. 12, 311 (2016).

[14] P. Dai, Antiferromagnetic Order, and Spin Dynamics in Iron-Based Superconductors, Rev. Mod. Phys. 87, 855 (2015).

[15] J. T. Park, G. Friemel, T. Loew, V. Hinkov, Y. Li, B. H. Min, D. L. Sun, A. Ivanov, A. Piovano, C. T. Lin, B. Keimer, Y.S. Kwon, and D. S. Inosov, Similar Zone-Center Gaps in the Low-Energy Spin-Wave Spectra of $\mathrm{Na}_{1-\delta} \mathrm{FeAs}$ and $\mathrm{BaFe}_{2} \mathrm{As}_{2}$, Phys. Rev. B 86, 024437 (2012).

[16] N. Qureshi, P. Steffens, S. Wurmehl, S. Aswartham, B. Büchner, and M. Braden, Local Magnetic Anisotropy in $\mathrm{BaFe}_{2} \mathrm{As}_{2}$ : A Polarized Inelastic Neutron Scattering Study, Phys. Rev. B 86, 060410(R) (2012).

[17] C. Wang, R. Zhang, F. Wang, H. Luo, L. P. Regnault, P. Dai, and Y. Li, Longitudinal Spin Excitations and Magnetic Anisotropy in Antiferromagnetically Ordered $\mathrm{BaFe}_{2} \mathrm{As}_{2}$, Phys. Rev. X 3, 041036 (2013).

[18] Y. Song, L.-P. Regnault, C. Zhang, G. Tan, S. V. Carr, S. Chi, A. D. Christianson, T. Xiang, and P. Dai, In-Plane Spin Excitation Anisotropy in the Paramagnetic State of NaFeAs, Phys. Rev. B 88, 134512 (2013).

[19] T. M. McQueen, A. J. Williams, P. W. Stephens, J. Tao, Y. Zhu, V. Ksenofontov, F. Casper, C. Felser, and R. J. Cava, Tetragonal-to-Orthorhombic Structural Phase Transition at $90 \mathrm{~K}$ in the Superconductor $\mathrm{Fe}_{1.01} \mathrm{Se}$, Phys. Rev. Lett. 103, 057002 (2009).

[20] Z. R. Ye, C. F. Zhang, H. L. Ning, W. Li, L. Chen, T. Jia, M. Hashimoto, D. H. Lu, Z.-X. Shen, and Y. Zhang, Simultaneous Emergence of Superconductivity, InterPocket Scattering and Nematic Fluctuation in PotassiumCoated FeSe Superconductor, arXiv:1512.02526.

[21] See Supplemental Material at http://link.aps.org/ supplemental/10.1103/PhysRevX.7.021025 for details of experimental methods, theoretical analyses, and additional measurement data.

[22] M. C. Rahn, R. A. Ewings, S. J. Sedlmaier, S. J. Clarke, and A. T. Boothroyd, Strong $(\pi, 0)$ Spin Fluctuations in $\beta-$ FeSe Observed by Neutron Spectroscopy, Phys. Rev. B 91, 180501 (2015).

[23] H. Luo, M. Wang, C. Zhang, X. Lu, L.-P. Regnault, R. Zhang, S. Li, J. Hu, and P. Dai, Spin Excitation Anisotropy as a Probe of Orbital Ordering in the Paramagnetic Tetragonal Phase of Superconducting $\mathrm{BaFe}_{1.904} \mathrm{Ni}_{0.096} \mathrm{As}_{2}$, Phys. Rev. Lett. 111, 107006 (2013).

[24] P. Steffens, C. H. Lee, N. Qureshi, K. Kihou, A. Iyo, H. Eisaki, and M. Braden, Splitting of Resonance Excitations 
in Nearly Optimally Doped $\mathrm{Ba}\left(\mathrm{Fe}_{0.94} \mathrm{Co}_{0.06}\right)_{2} \mathrm{As}_{2}$ : An Inelastic Neutron Scattering Study with Polarization Analysis, Phys. Rev. Lett. 110, 137001 (2013).

[25] C. Zhang, Y. Song, L.-P. Regnault, Y. Su, M. Enderle, J. Kulda, G. Tan, Z. C. Sims, T. Egami, Q. Si, and P. Dai, Anisotropic Neutron Spin Resonance in Underdoped Superconducting $\mathrm{NaFe}_{1-x} \mathrm{Co}_{x}$ As, Phys. Rev. B 90, 140502 (2014).

[26] O. J. Lipscombe, L. W. Harriger, P. G. Freeman, M. Enderle, C. Zhang, M. Wang, T. Egami, J. Hu, T. Xiang, M. R. Norman, and P. Dai, Anisotropic Neutron Spin Resonance in Superconducting $\mathrm{BaFe}_{1.9} \mathrm{Ni}_{0.1} \mathrm{As}_{2}$, Phys. Rev. B 82, 064515 (2010).

[27] C. Zhang, M. Liu, Y. Su, L.-P. Regnault, M. Wang, G. Tan, Th. Brückel, T. Egami, and P. Dai, Magnetic Anisotropy in Hole-Doped Superconducting $\mathrm{Ba}_{0.67} \mathrm{~K}_{0.33} \mathrm{Fe}_{2} \mathrm{As}_{2}$ Probed by Polarized Inelastic Neutron Scattering, Phys. Rev. B 87, 081101(R) (2013).

[28] N. Qureshi, C. H. Lee, K. Kihou, K. Schmalzl, P. Steffens, and M. Braden, Anisotropy of Incommensurate Magnetic Excitations in Slightly Overdoped $\mathrm{Ba}_{0.5} \mathrm{~K}_{0.5} \mathrm{Fe}_{2} \mathrm{As}_{2}$ Probed by Polarized Inelastic Neutron Scattering Experiments, Phys. Rev. B 90, 100502(R) (2014).

[29] F. Waßer, C. H. Lee, K. Kihou, P. Steffens, K. Schmalzl, N. Qureshi, and M. Braden, Suppression of Low-Energy Longitudinal Spin-Excitations in Co-Underdoped $\mathrm{BaFe}_{2} \mathrm{As}_{2}$, arXiv: 1609.02027.

[30] J.D. Wright, T. Lancaster, I. Franke, A. J. Steele, J. S. Möller, M. J. Pitcher, A. J. Corkett, D. R. Parker, D. G. Free, F. L. Pratt, P. J. Baker, S. J. Clarke, and S. J. Blundell, Gradual Destruction of Magnetism in the Superconducting Family $\mathrm{NaFe}_{1-x} \mathrm{Co}_{x}$ As, Phys. Rev. B 85, 054503 (2012).

[31] M. Liu, C. Lester, J. Kulda, X. Lu, H. Luo, M. Wang, S. M. Hayden, and P. Dai, Polarized Neutron Scattering Studies of Magnetic Excitations in Electron-Overdoped Superconducting $\mathrm{BaFe}_{1.85} \mathrm{Ni}_{0.15} \mathrm{As}_{2}$, Phys. Rev. B 85, 214516 (2012).

[32] D. S. Inosov, J. T. Park, A. Charnukha, Y. Li, A. V. Boris, B. Keimer, and V. Hinkov, Crossover from Weak to Strong Pairing in Unconventional Superconductors, Phys. Rev. B 83, 214520 (2011).

[33] C. Zhang, R. Yu, Y. Su, Y. Song, M. Wang, G. Tan, T. Egami, J. A. Fernandez-Baca, E. Faulhaber, Q. Si, and P. Dai, Measurement of a Double Neutron-Spin Resonance and an Anisotropic Energy Gap for Underdoped Superconducting $\mathrm{NaFe}_{0.985} \mathrm{Co}_{0.015}$ As Using Inelastic Neutron Scattering, Phys. Rev. Lett. 111, 207002 (2013).

[34] S. Kasahara, T. Watashige, T. Hanaguri, Y. Kohsaka, T. Yamashita, Y. Shimoyama, Y. Mizukami, R. Endo, H. Ikeda, K. Aoyama, T. Terashima, S. Uji, T. Wolf, H. von Löhneysen, T. Shibauchi, and Y. Matsuda, Field-Induced Superconducting Phase of FeSe in the BCS-BEC Crossover, Proc. Natl. Acad. Sci. U.S.A. 111, 16309 (2014).

[35] J.-Y. Lin, Y. S. Hsieh, D. A. Chareev, A. N. Vasiliev, Y. Parsons, and H.D. Yang, Coexistence of Isotropic and Extended s-Wave Order Parameters in FeSe as Revealed by Low-Temperature Specific Heat, Phys. Rev. B 84, 220507(R) (2011).

[36] M. Braden, P. Steffens, Y. Sidis, J. Kulda, P. Bourges, S. Hayden, N. Kikugawa, and Y. Maeno, Anisotropy of the
Incommensurate Fluctuations in $\mathrm{Sr}_{2} \mathrm{RuO}_{4}$ : A Study with Polarized Neutrons, Phys. Rev. Lett. 92, 097402 (2004).

[37] I. Eremin, D. Manske, and K. H. Bennemann, Electronic Theory for the Normal-State Spin Dynamics in $\mathrm{Sr}_{2} \mathrm{RuO}_{4}$ : Anisotropy due to Spin-Orbit Coupling, Phys. Rev. B 65 , 220502(R) (2002).

[38] A. Fedorov, A. Yaresko, T. K. Kim, Y. Kushnirenko, E. Haubold, T. Wolf, M. Hoesch, A. Grüneis, B. Büchner, and S. V. Borisenko, Effect of Nematic Ordering on Electronic Structure of FeSe, Sci. Rep. 6, 36834 (2016).

[39] M. M. Korshunov, Y. N. Togushova, I. Eremin, and P. J. Hirschfeld, Spin-Orbit Coupling in Fe-Based Superconductors, J. Supercond. Novel Magn. 26, 2873 (2013).

[40] P. Zhang, T. Qian, P. Richard, X. P. Wang, H. Miao, B. Q. Lv, B. B. Fu, T. Wolf, C. Meingast, X. X. Wu, Z. Q. Wang, J. P. Hu, and H. Ding, Observation of Two Distinct $d_{x z} / d_{y z}$ Band Splittings in FeSe, Phys. Rev. B 91, 214503 (2015).

[41] P. Babkevich, B. Roessli, S. N. Gvasaliya, L.-P. Regnault, P. G. Freeman, E. Pomjakushina, K. Conder, and A. T. Boothroyd, Spin Anisotropy of the Resonance Peak in Superconducting $\mathrm{FeSe}_{0.5} \mathrm{Te}_{0.5}$, Phys. Rev. B 83, 180506(R) (2011).

[42] K. Prokeš, A. Hiess, W. Bao, E. Wheeler, S. Landsgesell, and D. N. Argyriou, Anisotropy of the $(\pi, \pi)$ Dynamic Susceptibility in Magnetically Ordered $(x=0.05)$ and Superconducting $(x=0.40) \mathrm{Fe}_{1.02} \mathrm{Te}_{1-x} \mathrm{Se}_{x}$, Phys. Rev. B 86, 064503 (2012).

[43] P. S. Wang, S. S. Sun, Y. Cui, W. H. Song, T. R. Li, R. Yu, H. Lei, and W. Yu, Pressure Induced Stripe-Order Antiferromagnetism and First-Order Phase Transition in FeSe, Phys. Rev. Lett. 117, 237001 (2016).

[44] F. Waßer, A. Schneidewind, Y. Sidis, S. Wurmehl, S. Aswartham, B. Büchner, and M. Braden, Spin Reorientation in $\mathrm{Ba}_{0.65} \mathrm{Na}_{0.35} \mathrm{Fe}_{2} \mathrm{As}_{2}$ Studied by Single-Crystal Neutron Diffraction, Phys. Rev. B 91, 060505(R) (2015).

[45] J. M. Allred, K. M. Taddei, D. E. Bugaris, M. J. Krogstad, S. H. Lapidus, D. Y. Chung, H. Claus, M. G. Kanatzidis, D. E. Brown, J. Kang, R. M. Fernandes, I. Eremin, S. Rosenkranz, O. Chmaissem, and R. Osborn, Double- $Q$ Spin-Density Wave in Iron Arsenide Superconductors, Nat. Phys. 12, 493 (2016).

[46] V. Cvetkovic and O. Vafek, Space Group Symmetry, SpinOrbit Coupling, and the Low-Energy Effective Hamiltonian for Iron-Based Superconductors, Phys. Rev. B 88, 134510 (2013).

[47] M. H. Christensen, J. Kang, B. M. Andersen, I. Eremin, and R. M. Fernandes, Spin Reorientation Driven by the Interplay between Spin-Orbit Coupling and Hund's Rule Coupling in Iron Pnictides, Phys. Rev. B 92, 214509 (2015).

[48] N. F. Q. Yuan, K. F. Mak, and K. T. Law, Possible Topological Superconducting Phases of $\mathrm{MoS}_{2}$, Phys. Rev. Lett. 113, 097001 (2014).

[49] J. M. Lu, O. Zheliuk, I. Leermakers, N. F. Q. Yuan, U. Zeitler, K. T. Law, and J. T. Ye, Evidence for TwoDimensional Ising Superconductivity in Gated $\mathrm{MoS}_{2}$, Science 350, 1353 (2015).

[50] J. L. Her, Y. Kohama, Y. H. Matsuda, K. Kindo, W.-H. Yang, D. A. Chareev, E. S. Mitrofanova, O. S. Volkova, A. N. Vasiliev, and J.-Y. Lin, Anisotropy in the Upper Critical Field of FeSe and $\mathrm{FeSe}_{0.33} \mathrm{Te}_{0.67}$ Single Crystals, Supercond. Sci. Technol. 28, 045013 (2015). 